ARCHIVO ESPAÑOL DE ARTE, LXXXI, 322

ABRIL-JUNIO 2008, pp. 105-114

ISSN: 0004-0428

\title{
UN NUEVO CATÁLOGO PICTÓRICO DEL QUATTROCENTO ITALIANO: LA TABLA DE CAMERINO Y EL DESAPARECIDO CICLO JACOBEO DE GIOVENALE DE ORVIETO EN ARACELI*
}

\author{
POR \\ Rosa VÁzQuez SANTOS \\ Investigadora Parga Pondal de la S. A. de Xestión do Plan Xacobeo
}

\begin{abstract}
El presente trabajo sugiere una solución conjunta a dos problemas: la atribución de una tabla del Museo Diocesano de Camerino y la hagiografía del misterioso "milagro del caballero cubierto de conchas" en ella representado. Las fuentes documentales relacionadas con la presencia de arte, culto e iconografía de Santiago el Mayor en la ciudad de Roma, principalmente la descriptiva obra de Miguel Erce Jiménez, nos permiten relacionar dicha tabla con el desaparecido retablo de Santa Maria de Araceli del pintor Giovenale de Orvieto, inaugurando el catálogo del artista. Al mismo tiempo las fuentes citadas nos llevan al origen portugués de la iconografía del milagro.
\end{abstract}

Palabras clave: Iconografía jacobea; Milagro; Camerino; La Spezia; Roma; Santa Maria Araceli; Giovenale de Orvieto; Miguel Erce Jiménez.

This study proposes a combined solution to two problems: the attribution of an altar panel from the Museo Diocesano of Camerino and the hagiography of the mysterious "miracle of the knight covered with shells" in this painting. The documentary sources related to the art, cult and iconography of Saint James the Great in Rome, in particular the descriptive work of Miguel Erce Jiménez, allow us to connect this panel with the lost altarpiece in Santa Maria Araceli by Giovenale of Orvieto, thus becoming the first picture in his catalogue. At the same time, these sources lead us to the Portuguese origin of the iconography of this miracle.

Key words: Iconography of St. James; Miracle; Camerino; La Spezia; Rome; Santa Maria Araceli; Giovenale of Orvieto; Miguel Erce Jiménez.

* El presente artículo es fruto de mis años de investigación en Roma y Perugia como becaria postdoctoral del MECD y del MAE-AECI. Quiero hacer constar mi agradecimiento a Paolo Caucci von Saucken y Humbert Jacomet por su colaboración, a Elvio Lunghi por las primeras referencias sobre Giovenale de Orvieto y al personal de la American Academy y de la Biblioteca Hertziana de Roma, cuya amabilidad y eficacia han ayudado tanto a mis investigaciones. 
A lo largo de este artículo recorreremos brevemente la historia de dos problemas que, finalmente, convergerán en una solución conjunta: uno de carácter iconográfico y estilístico y otro referido al catálogo del pintor Giovenale de Orvieto, hasta ahora inexistente.

\section{La tabla de Camerino: problemas iconográficos y de atribución}

El primero de los problemas surgió en 1992, cuando el profesor Serafín Moralejo coordinó en Santiago de Compostela la exposición "Santiago Camino de Europa". En dicha muestra se exhibió una pequeña tabla pintada $(37,2 \times 77,7 \mathrm{~cm}$.) procedente del Museo Diocesano de Camerino, en Las Marcas, en la que convivían varios episodios de la leyenda de Santiago el Mayor ${ }^{2}$. Dos de las escenas de dicha tabla representan los sucesos más célebres de la traslación marítima y terrestre del Apóstol, remitiéndonos a las fuentes más convencionales: el texto de Jacopo Varazze y el Liber Sancti Iacobi o Codex Calixtinus ${ }^{3}$. Junto a estos episodios comparecía un enigmático caballero saliendo del mar con su cuerpo y el de su caballo totalmente cubiertos de conchas, figura a la que Lucia Gai intentó relacionar con algunos de los milagros atribuidos al Santo ${ }^{4}$. Posteriormente Humbert Jacomet publicó otra representación que parecía seguir la misma fuente literaria: una miniatura procedente de un códice del siglo XIV conservado en el Musée Condé de Chantilly 5 .

Desde una perspectiva estilística la tabla despertó también el interés de varios historiadores del arte. Federico Zeri la relacionó con otras dos: una conservada en el Museo Amedeo Lia de La Spezia $(36,5 \times 46 \mathrm{~cm}$.) y otra perteneciente a una colección privada de Pau, atribuyéndolas al pintor Antonio Alberti da Ferrara (1420-1442) ${ }^{6}$. Años después la monografía "Il Quattrocento a Camerino", coordinada por Andrea De Marchi, volvió a relacionar las tablas de Camerino y La Spezia, aunque en este caso la atribución recayó en el pintor Giacomo di Nicola ${ }^{7}$.

${ }^{1}$ S. Moralejo (Coord.): Santiago Camino de Europa. Culto y cultura de peregrinación a Compostela, Xunta de Galicia, Santiago de Compostela, 1993.

2 L. GaI: "Tabla del retablo de Camerino con la representación de la traslatio a Santiago", en S. MoraleJo (Coord.): Santiago Camino de Europa, Xunta de Galicia, Santiago de Compostela, 1993, pp. 499-500.

${ }^{3}$ No es este el lugar para introducir una nota historiográfica completa sobre las diferentes ediciones de ambas fuentes, por lo que me limitaré a citar las ediciones más recientes: Liber Sancti Jacobi. Codex Calixtinus, transcripción a partir del códice original por K. Herbers-M. Santos Noia, Xunta de Galicia, Santiago de Compostela, 1998 y I. DA Varazze, Legenda Aurea, edición crítica a cargo de G. P. Maggioni, Sismel, Edizioni del Galluzzo (2ª Ed.), Firenze, 1998, vol. 1, pp. 650-662. Entre las traducciones me remitiré a la castellana de Moralejo, Torres y Feo que, en su reciente reedición de 2004, aporta una bibliografía actualizada: Liber Sancti Jacobi. Codex Calixtino, traducción por A. Moralejo-C. Torres-J. Feo en la edición revisada por J. J. Moralejo-M. J. García Blanco, Xunta de Galicia, Santiago de Compostela, 2004.

${ }_{4}$ Particularmente con el milagro noveno del Codex Calixtinus, ocurrido a un joven cruzado francés salvado por Santiago de una tempestad mientras regresaba en barco de Jerusalén (LSI (Ed.), Lib. II, Cap. IX, f. 147r-v; LSI (Trad.), pp. 349-50).

${ }^{5}$ Nuevamente aparecen representados un caballero y su caballo que emergen del mar cubiertos de conchas $(\mathrm{H}$. JACOMET: "Une géographie des miracles de Saint Jacques propre à l'arc méditerrenèen", en P. CAUCCI vON SAUCKEN (Coord.): Santiago e l'Italia, p. 346, fig. 28).

${ }^{6}$ F. Zeri: "Antonio Alberti da Ferrara. Miracolo di san Giacomo maggiore", en I cataloghi del Museo Civico Amedeo Lia. 3. Dipinti, Silvana Editoriale, Milano, 1997, pp. 25-26.

7 M. Mazzalupi, "Giacomo di Nicola. Traslazione del corpo di san Giacomo Maggiore", en A. DE MarChI - M. GiAnNATEMPo LóPez (Coord.): Il Quattrocento a Camerino. Luce e prospettiva nel cuore della Marca, Federico Motta Editore, Milano, 2002, pp. 170-171. 


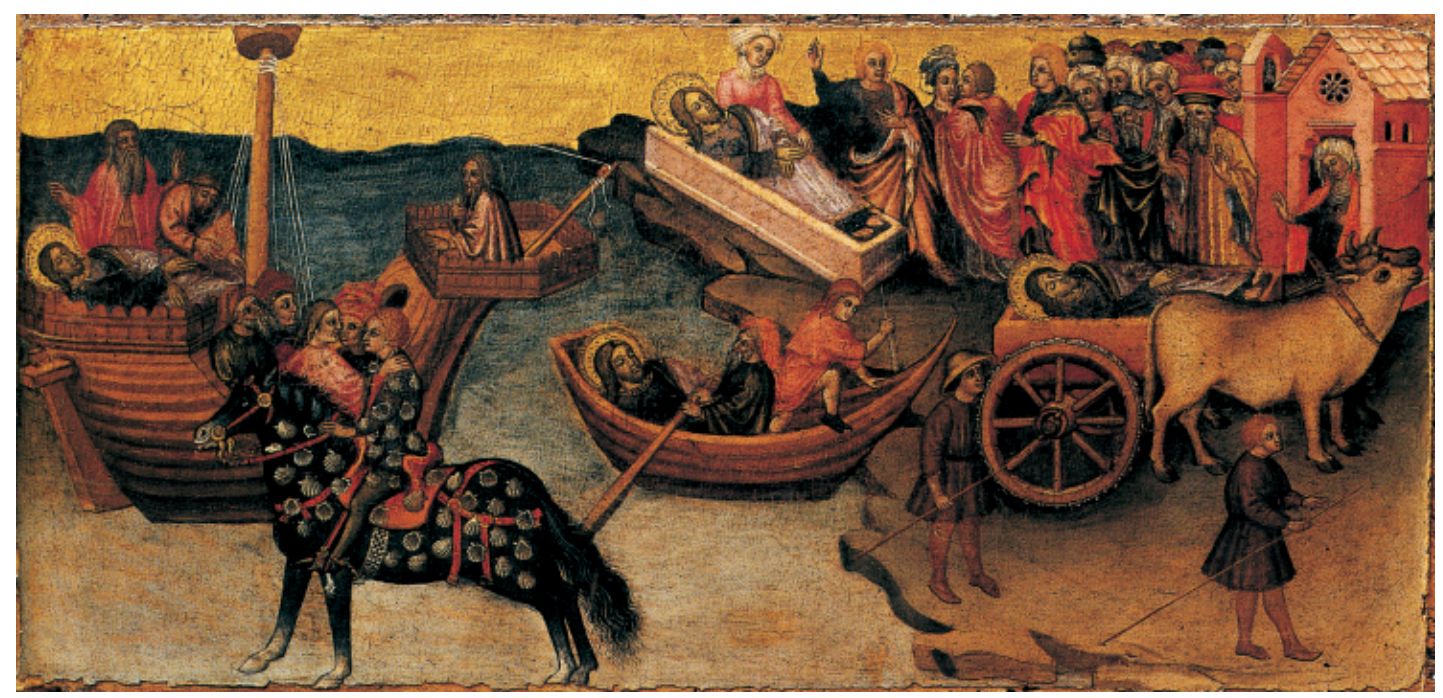

Fig. 1. Giovenale de Orvieto. Milagros de Santiago el Mayor. 1441. Museo Diocesano de Camerino. Procedente de la iglesia de Santa Maria Araceli de Roma.

\section{Giovenale de Orvieto y la capilla de San Giacomo de Araceli en Roma}

El segundo misterio se presentó durante mis investigaciones sobre el culto y la iconografía jacobeos en la ciudad de Roma. Desde el siglo XIV, guías e historias de la ciudad citaban la existencia de una Cappella di San Giacomo en la iglesia de Araceli en el lugar que actualmente ocupa la de San Michele Arcangelo ${ }^{8}$.

Gracias a Casimiro ${ }^{9}$ y, como siempre, a la monumental obra de Forcella ${ }^{10}$, se conocía la existencia en la capilla de una pala d'altare pintada en 1441 por Giovenale de Orvieto. Hoy sabemos que dicha inscripción se disponía sobre el propio retablo, escrita en letras doradas ${ }^{11}$, y que por tanto se perdió cuando aquél fue desmontado y vendido, no como consecuencia de la "violenta" reforma que la iglesia sufrió en tiempos del papa Pío IV (1559-1565) ${ }^{12}$.

\footnotetext{
${ }^{8}$ Fundada en el Trecento, mantuvo su advocación hasta los primeros años del siglo XIX (M. CARTA y L. Russo, S. María in Aracoeli, Le Chiese di Roma Illustrate, Roma, 1988, pp. 125-128).

${ }^{9}$ Casimiro publicó por primera vez una inscripción existente en el altar que, a su vez, había copiado de Aymaden: HOC OPVS FECIT FIERI NOBILIS VIR LAVRÊNTIVS PETRI OIA SCI / ALIAS DICTVS MANCINO DE LVCIIS TPE DNI EVGENII PP. IV. / M. CCCCXLI. IVVENALIS DE URBE VETERI M. SP. XII (F. CASIMIRO ROMANO, Memorie istoriche delle chiese e dei conventi dei Frati Minori della provincia Romana. Roma, 1845, m. 5671, p. 192).

${ }^{10}$ Forcella reproduce el texto de Casimiro en el capítulo que dedica a Santa Maria Araceli, si bien considera que se trata de una reproducción "infedelissima e pesima" (V. ForCELla, Iscrizioni delle chiese e d'altri edifici di Roma. Dal secolo XI fino ai giorni nostri. 14 vol., Roma, 1869-84, I, p. 136).

11 "ai á la parte del Evangelio una Capilla mui capaz tercera en orden, dedicada à Santiago el Zedebeo con esta inscripción entre el Retablo, i su vasa: Hoc opus fecit fieri nobilis vir Laurentius Petronnia Sancti, alias dictus Mancino de Lutÿs tempore Domini Eugenÿ Papa IV. anno M.CD.XLI. Iuvenalis de urbeveteri me pixit” (M. DE ERCE Y JIMÉNEZ, Prueva evidente de la predicación del Apóstol Santiago el Mayor en los reinos de España. Madrid, Alonso de Paredes, 1644, Parte II, Trat. II, Cap. I, p. 229, 4).

12 Hipótesis citada por Forcella (V. Forcella, Iscrizioni cit., I, p. 135).
} 


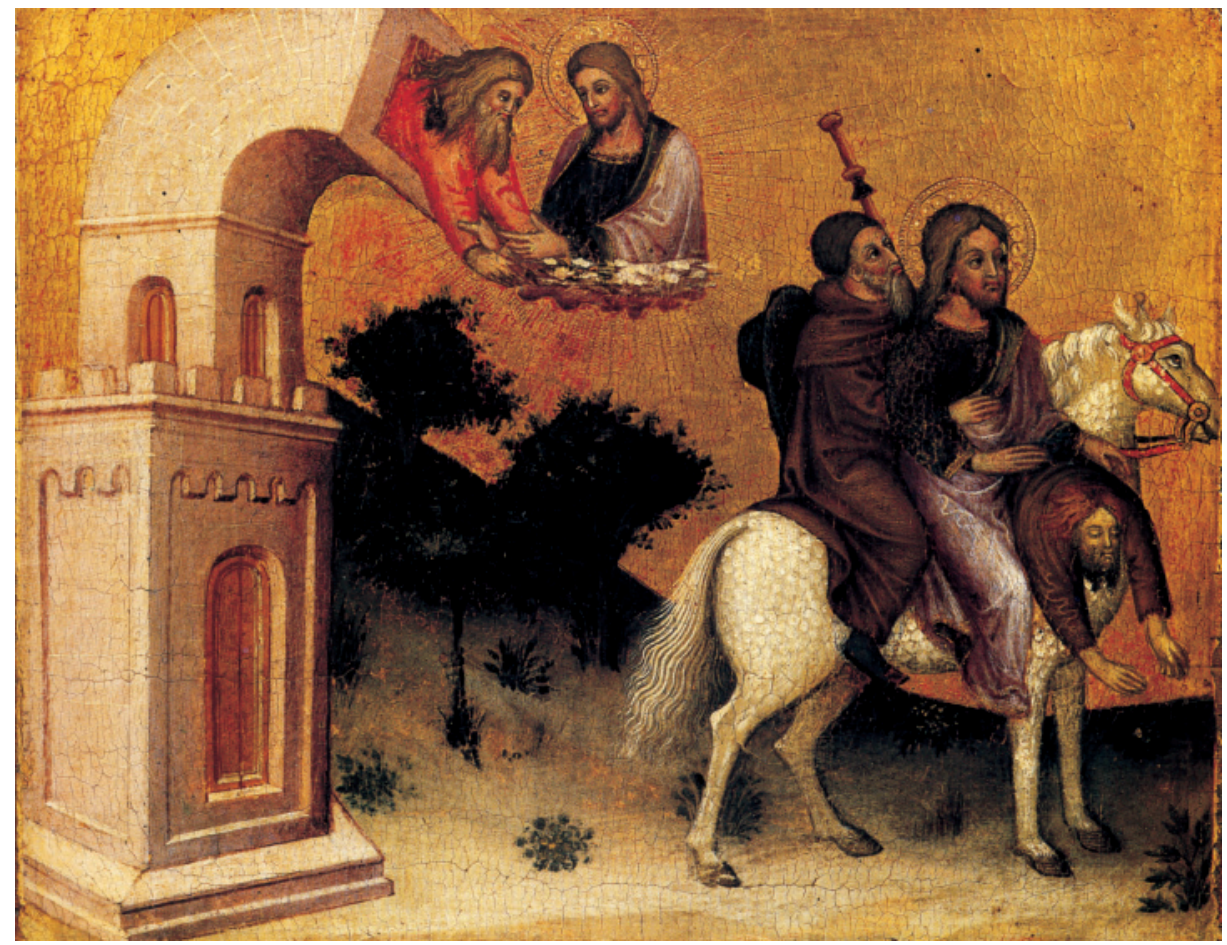

Fig. 2. Giovenale de Orvieto. Milagros de Santiago el Mayor. 1441. Museo Civico Amedeo Lia de La Spezia. Procedente de la iglesia de Santa Maria Araceli de Roma.

La obra había sido comisionada por un cierto Manzino de Lutÿs de la familia Mancini o Massimi, protagonista de una de las principales ascensiones sociales de la Roma del siglo $\mathrm{XV}^{13}$. Sin embargo, el programa iconográfico del altar se ignoraba por completo, hasta el punto de estar ausente de las guías de Roma escritas con anterioridad a la reforma de la capilla y desaparición del altar, incluso de la de Celio, la más rica en datos artísticos ${ }^{14}$. Esta carencia de datos no es extraña pues, a pesar de su fama como uno de los principales pintores de su tiempo, favorito del papa Nicolò V (1447-55), se desconoce casi todo del Giovenale de Orvieto y sus obras ${ }^{15}$, estando todavía hoy pendiente la elaboración de un catálogo propio ${ }^{16}$.

${ }^{13}$ En su trabajo sobre la nobleza romana del período Modigliani documenta a un cierto "Iacobus Mancini de Lutiis", patriarca de la familia que por su patronímico podría haber sido el fundador de la capilla (A. ModigLIANI, "Li Nobili Huomini di Roma”, en S. Gensini (Coord.): Roma Capitale (1447-1527), Pubblicazioni degli Archivi di Stato, Saggi 29, pp. 356, 357 y n. 37). Dicho comportamiento sería completamente normal en un mundo donde una familia como la Della Valle utilizó como señal de ascenso social el paso de su capilla desde la iglesia de $S$. Lorenzo in Ascesa a otra como S. Maria dell'Araceli (Ibidem, p. 376).

${ }^{14}$ G. Celio, Memoria delli nomi dell'Artefici delle Pitture, che sono in alcune Chiese, Facciate, Palazzi di Roma. Napoli, Scipione Bonino, 1638, p. 54. El silencio se extiende a las "vite" que, empezando por el Vasari, ignoran completamente al pintor (G. VASARI, Le vite de' più eccellenti pittori scultori e architettori. Nelle relazioni del 1550 e 1568 , testo a cura di Rossana Bettarini e commento secolare a cura di Paola Barocchi, Firenze, 1967, 6 vol.).

15 Apenas ocupa espacio en la bibliografía artística: ALLGEMEINES LEXIKON DER BILDENDEN KÜNSTLER, Herausgegeben von ULRICH THIEME UND FRED. C. WILLIS, Vierzehnter Band, Veb E.A.Seemann Verlag Leipzig, 1921, v. XIV, p. 151; DIZIONARIO ENCICLOPÉDICO BOLAFFI DEI PITTORI E DEGLI INCISORI ITALIANI, coord. por A. Bolaffi-U. Allemandi, Torino, 1972-76, VI, p. 72; H. GNoli: Pittori e miniatori nell'Umbria, Ediclio, Spoleto, 


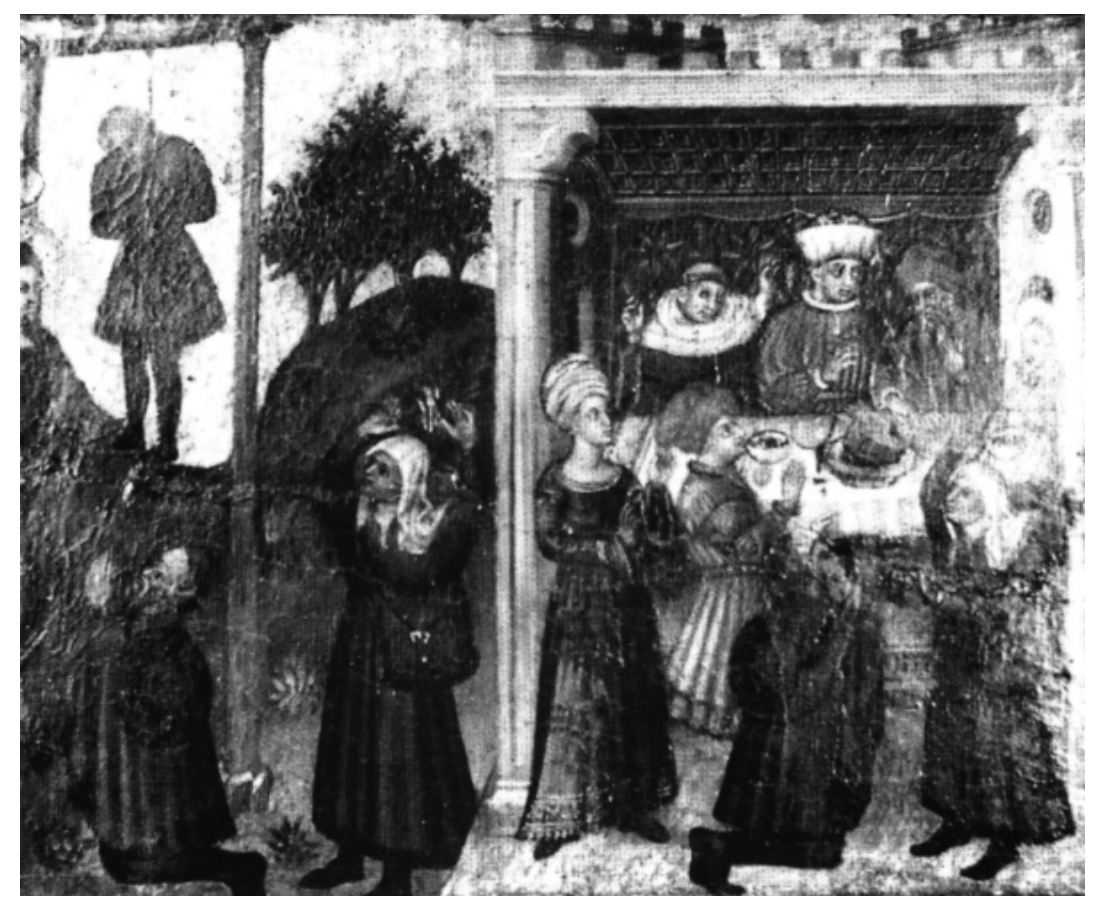

Fig. 3. Giovenale de Orvieto. Milagros de Santiago el Mayor. 1441. Colección privada de Pau. Procedente de la iglesia de Santa Maria Araceli de Roma.

Las noticias sobre la capilla son más numerosas en el siglo XVII, existiendo un inventario de 1662 que alude a la capilla "Di s. Giacomo Apostolo, fondata dagli Ss. Lucci Manzini"17. Pero la mayor parte de las noticias proceden de la reforma emprendida por Francesco Maria Manzini, nombrado cardenal por el papa Alessandro VII (1655-67) en 1660, quien dotó la obra a su muerte, en 1672, aunque no como capilla funeraria, pues fue enterrado en la iglesia de San Barnaba $^{18}$. Se sustituyó entonces la obra del pintor de Orvieto por un altar pintado de Giovanni Battista Buoncuore en el que, según diferentes fuentes, estaban representados Santiago el Mayor, san Estéfano y san Hipólito ${ }^{19}$ o Santiago el Mayor y san Lorenzo ${ }^{20}$.

1923, pp. 169-70; DE Nicola: "Il tesoro di San Giovanni in Laterano fino al secolo XV", en Bolletino d'Arte, 1909, p. 41 notas 1 y 5.

${ }^{16}$ De todas las fuentes referidas en la nota 15 solamente el diccionario Bolaffi atribuye una obra al pintor, atribución muy discutible (DIZIONARIO cit., VI, p. 72).

17 M. Armellini, Chiese di Roma dal secolo IV al XIX, a cura di Carlo CeCChelli, tomo I, Roma 1942, p. 545.

18 G. SiCARI, Cenni biografici su tutti i Cardinali (1198-2001), Roma, 2001, p. 66.

19 Es Titi quien, en su célebre guía de 1674, informa de la obra apenas ejecutada: "Passata la Cappella contigua, vi è quella dedicata à S. Giacomo, quale si vede effigiato nel Quadro con S. Stefano, e S. Lorenzo con buona maniera da Gio: Battista Buoncore da Ascoli" (F. TITI, Studio di Pittura, Scoltura, et Architettura, nelle Chiese di Roma, Edizione comparata a cura di Bruno Contardi e Serena Romano, 1987, p. 110).

20 C. Santucci, "Vita di Giambatista Boncuore", en L. Pascoli, Vite de' pittori, scultori, ed architetti moderni, Edizione critica dedicata a Valentino Martinelli, Electa Editori Umbri, Perugia, 1992, vol. II, p. 718. 


\section{Una solución conjunta a dos problemas: La Prueva Evidente de Don Miguel de Erce Ximénez}

Los dos problemas expuestos, surgidos en momentos y lugares diferentes, han terminado por resolverse de forma inesperada y conjunta gracias a una obra del siglo XVII: un extenso texto que Miguel de Erce y Jiménez dedicó al retablo de Giovenale de Orvieto en 1644.

La vida y hechos del que fuera canónigo de las catedrales de León y Santiago de Compostela y, posteriormente, capellán del rey en la "Real" de Toledo, está recogida en una inscripción del altar de la capilla del Cristo de la parroquia de San Esteban de Préjano (Logroño) ${ }^{21}$. Como escritor dedicó un enorme esfuerzo al estudio de la leyenda y culto jacobeo del que nació su monumental Prueva evidente de la predicación del Apóstol Santiago el Mayor en los Reinos de España ${ }^{22}$, obra que responde al mismo espíritu apologético que las más conocidas de $\mathrm{Oxea}^{23}$ y Castellá Ferrer ${ }^{24}$. Inesperadamente, la obra ofrece un verdadero tesoro para el investigador jacobeo en Roma, al dedicar varias páginas al culto y la iconografía de Santiago en la ciudad donde el propio Erce y Jiménez había vivido durante años ${ }^{25}$.

Según la minuciosa descripción de Erce, el retablo de Araceli era de madera dorada y en su cuerpo principal contaba con las imágenes de Santiago el Mayor, con libro y bordón, y, en menor tamaño, las de san Esteban y san Lorenzo ${ }^{26}$.

La predella o banco estaba compuesta por tres tablas, de las cuales la central poseía mayor tamaño y número de escenas. En su parte izquierda se agrupaban las representaciones de la traslación marítima del Apóstol desde Jafa a tierras de Galicia ${ }^{27}$ y la enigmática figura del caballero y su caba-

21 "Este santo Cristo, con cuatro cuadros de la historia de Santiago y otro de la Virgen del Popolo para adorno de esta capilla, diò de limosna á esta iglesia año de 1648 el Dr. D. Miguel de Herce Jiménez, natural de su real capilla de los Reyes nuevos de Toledo, donde murió, electo obispo de Tuy á 13 de septiembre de 1648 y fue quien por su grande estudio y diligencia, y à su costa, hizo poner la afirmativa en el Breviario, de que el Sr. Santiago el Mayor predicó en España. Y este retablo y reparo de esta capilla se hizo à su costa y en este altar dejò fundada una misa todas las fiestas del año á las once horas del día. Acabóse este altar en 18 de julio de 1649” (V. Herrero Mediavilla-R. Aguayo NAYLE, Archivo biográfico de España e Iberoamérica, Müncher: Saur, 1986, I, ficha 436, pp. 27-28).

22 M. De Erce Ximénez, Prueva evidente de la predicación del Apóstol el Mayor en los Reinos de España, Alonso de Paredes, Madrid, 1644.

${ }^{23}$ H. OXEA, Historia del Glorioso Apóstol Santiago Patrón de España, de su venida a ella, y de las grandezas de sus iglesias y órdenes militares, Madrid, 1615.

${ }^{24}$ M. Castellá Ferrer, Historia del Apóstol de Iesvs Christo, Sanctiago Zebedeo, Patrón y Capitán General de las Españas, Santiago de Compostela, Madrid, 1610, ed. facsímil 2000.

${ }^{25}$ La presencia del autor en Roma queda patente en las diferentes descripciones presentes en la segunda parte del libro que, sin duda, buscan reforzar el poder español en la ciudad cuando la disminución de las relaciones entre España el papado resultaban evidentes pues, tras su elección, el papa Urbano VIII había iniciado la construcción de un conjunto de fortificaciones hasta entonces prohibidas por un tratado firmado entre el papa Pablo IV y Felipe II en 1557 [T. J. DAndelet, La Roma española (1500-1700). Barcelona, 2002, p. 233]. En el presente artículo nos hemos limitado a las páginas que Erce dedica al retablo en el Apéndice I, el texto dedicado al culto y presencia jacobea en la ciudad de Roma será objeto de otros dos artículos de próxima publicación.

26 "El Retablo es de madera dorada, la obra rica. Está Santiago en medio del Retablo cuerpo entero en pie, $i$ rostro tan hermoso, que parece el Salvador, la barba abierta, bordon en la mano derecha, i libro en la otra. A los dos lados S. Estevan, i S. Lorenço cuerpos enteros algo mas pequeños, i encima en la cornisa, que forma el quadro del Retablo tres cabeças de hermosos Cherubines en correspondencia destos tres cuerpos. En la parte superior dél ai un Christo en la Cruz con la Virgen, i S. Juan à los lados. I en cada una de las extremidades fuera del cuerpo del Retablo ai tres Santos desde el asiento del Retablo hasta arriba” (M. DE ERCE Y JIMÉNEZ, Prueva evidente cit., Parte II, Trat. II, Cap. I, p. 229, 5).

27 "La vasa está dividida en tres partes, en la de medio está pintada una nave en el mar, $i$ en ella el cuerpo de Santiago sobre la popa rostro venerable abierta la barba. También ai dentro de la nave siete hombres, que son los Dis- 
llo totalmente cubiertos de conchas ${ }^{28}$, mientras que en su parte central y derecha se representaban tres episodios relativos a la traslación terrestre: la llegada de la barca a Iria Flavia ${ }^{29}$, el prodigio del arca de piedra ${ }^{30}$ y la llegada al palacio de la reina Lupa tras la milagrosa doma de los toros ${ }^{31}$.

Las dos tablas laterales eran de menor tamaño y agrupaban solamente dos escenas en cada una, todas ellas relativas a tres de los milagros incluidos tanto en el Liber Sancti Jacobi como en La Leyenda Dorada. En la tabla del lado del Evangelio estaban representados el milagro del comerciante encerrado en una torre ${ }^{32}$ y el conocido como de los treinta peregrinos loreneses $^{33}$. En la tabla del lado de la Epístola el famoso milagro del peregrino ahorcado estaba desarrollado en dos escenas ${ }^{34}$, incorporando la segunda de ellas el episodio del gallo y la gallina que cantaron después de $\operatorname{asados}^{35}$.

cípulos, que vinieron de Ierusalen a España en custodia del santo cuerpo, luego que el Rei Herodes le hizo degollar" (M. De ERce y Jiménez, Prueva evidente cit., Parte II, Trat. II, Cap. I, p. 229, 6). Como fuentes iconográficas véase: I. DA VARAZZe, v. 1, p. 654; $L S J$ (ed.), Lib. I, Cap. 9, f. 52v; $L S J$ (ed.), Lib. I, Cap. 9, f. 157r.

28 "Prosiguiendo, pues, la relacion del Retablo de nuestro Apóstol, digo, que junto a la nave sobre el mismo mar ai un Cavallero en un cavallo negro con silla, i adorno rojo rico cubriendo todo su cuerpo, i el del cavallo de conchas mui perfectas pintadas de la haz, i enves. Està hablando con los de la nave echado el braço por el cuello de uno como sustentandose en el" (M. DE ERCE Y JiMÉNEZ, Prueva evidente cit., Parte II, Trat. II, Cap. I, p. 230, 7). Los problemas de la hagiografía de este milagro serán tratados más adelante.

29 "cerca del milagro de las Conchas, sobre que hemos discurrido, ai una barquilla guiada de dos remeros tocando con la popa en tierra, i dentro della el cuerpo de Santiago en la forma, que en la nave, de donde se supone, que lo sacavan con la barquilla para tomar tierra en Iria Flavia (oi el Padron) Ciudad, i Puerto de Galicia, adonde fue a parar la nave desde Jope" (M. DE ERCE y JimÉneZ, Prueva evidente cit., Parte II, Trat. II, Cap. I, p. 232, 25. Fuentes iconográficas: I. DA VARAZZE, p. 654 y LSJ (ed.), Lib. I, Cap. 9, f. 157v).

30 "Mas arriba està el cuerpo dentro de una piedra grande en forma de sepulcro: i es caso, que aviendole puesto sobre un marmol à la lengua del agua, en tomando tierra, se ablandò como cera, i hecho sepulcro abraçò en si al santo cuerpo" M. DE ERCE y JimÉnEZ, Prueva evidente cit., Parte II, Trat. II, Cap. I, p. 232, 26. Fuentes iconográficas: I. DA VARAZZE, p. 654 y $L S J$ (ed.), Lib. I, Cap. 9, f. 157v.

31 "Poco mas abaxo, $i$ adelante estan dos bueyes tirando un carro, $i$ dentro del la piedra con el cuerpo del Apostol (de suerte, que en sola esta division de la basa del Retablo està pintado en quatro partes el cuerpo entero con efigie hermosa, $i$ venerable) los bueyes entran en la casa de la Reina, la qual està à las ventanas admirandose dello, $i$ con razon, porque es uno de los mayores milagros de Santiago" (M. DE ERCE Y JiMÉNEZ, Prueva evidente cit., Parte II, Trat. II, Cap. I, p. 232, 27). Fuentes iconográficas: I. DA VARAZZE, v. 1, p. 655 y LSJ (ed.), Lib. I, Cap. 9, f. 158v-159r.

32 "En la otra division à la parte del Evangelio està pintada à la mano derecha una torre con la estremidad inclina$d a$, de suerte que se haze un arco, i està Santiago en tierra echando los braços a uno, que sale della. Dicen los Autores desta historia, i especialmente Calisto II en el lib. 2. que trata los milagros del Apostol, que era un mercader, à quien cierto señor tirano avia quitado su hazienda, i encarcelado en aquella torre, i que encomendansose à Santiago se inclinò la torre hasta la tierra, i salio libre el preso à pie llano" (M. DE ERCE Y JIMÉNEZ, Prueva evidente cit., Parte II, Trat. II, Cap. I, p. 233, 29). Fuentes iconográficas: I. DA VARAZZE, v. 1, pp. 659-60 y LSJ (ed.), Lib. II, Cap. 14, f. 148v.

33 "A la mano izquierda està pintado Santiago en un cavallo blanco con un hombre muerto al razón de la silla, $i$ à las ancas un peregrino con su bordon. El muerto es uno de treinta Loreneses, que ivan a Compostela en compañia jurada, $i$ aviendole desamparado en la enfermedad los demas, $i$ asistiendole uno, que no era de la compañia, muerto el enfermo invocò el peregrino, que le assistia, à Santiago, $i$ vino, i les llevò aquella noche quinze jornadas...” (M. DE ERCE y JimÉnez, Prueva evidente cit., Parte II, Trat. II, Cap. I, p. 233, 30). Fuentes iconográficas: I. DA VARAZZE, v. 1, p. 656 y $L S J$ (ed.), Lib. II, Cap. 4, f. 143r-144r.

34 "De la parte de la Epistola à la mano derecha del tercer apartamento, i division està colgado de la horca un peregrino moço vivo, que yendo en peregrinacion à Compostela le ahorcaron injustamente en Santo Domingo de la Calçada ( $i$ no en Tolosa, como por error dicen algunos) i Santiago està à su lado sustentándole con ambas manos por los pies, para que no padeciesse. El padre del moço està de rodillas puestas las manos mirando al Apostol, i à su hijo, i la madre en pie con los braços abiertos levantados admirada, i alegre de ver vivo al hijo despues de treinta, i seis dias, que le avian ahorcado, en los quales fueron, i bolvieron de Santiago" (M. DE ERCE Y JiMÉNEZ, Prueva evidente cit., Parte II, Trat. II, Cap. I, p. 233, 31). Fuentes iconográficas: I. DA VARAZZE, v. 1, p. 657 y LSJ (ed.), Lib. II, Cap. 5, f. 144v-145r.

35 M. De Erce y Jiménez, Prueva evidente cit., Parte II, Trat. II, Cap. I, p. 233, 32. El episodio del gallo y la gallina que cantaron después de muertos no figura en las fuentes más antiguas ni en los "exemplum" de los Frailes Predicadores, 
La descripción que Erce ofrece del retablo es realmente pormenorizada, deteniéndose en los problemas hagiográficos y las fuentes de cada uno de los milagros, particularmente en el misterioso episodio del caballero cubierto de conchas $^{36}$. En este sentido el texto contribuye en gran medida a la resolución del problema iconográfico que la escena planteaba, al citar varias fuentes hagiográficas, muchas de ellas tomadas de las obras, ya mencionadas, de Castellà Ferrer y $\mathrm{Oxea}^{37}$. Ambos autores se refieren al milagro relacionándolo con la fundación de una dinastía y su escudo de armas, pero si Castellá Ferrer identifica al protagonista del milagro con el hijo de un rey de Portugal y el emblema con el blasón del apellido "vieira" ${ }^{38}$, Oxea busca su origen en un milagro similar ocurrido en un río cercano a Santiago ${ }^{39}$, citado también por el Licenciado Molina en relación con el apellido y escudo de los Pimentel ${ }^{40}$.

Siguiendo las fuentes más antiguas hemos encontrado la solución en los estudios de antropología e historia de la devoción popular en Portugal, concretamente en los trabajos sobre la traslación de Santiago y el origen y significado de la concha o "vieira" de Mário Martins ${ }^{41}$ y Arlindo de Magalhães ${ }^{42}$. Los dos autores se ocupan de la leyenda jacobea del "filho de algum regulo que senhoreava a Maya", leyenda que explica la iconografía del caballero que emergió del mar cubierto de conchas ${ }^{43}$ y tiene una de sus fuentes más antiguas en un códice del monasterio de Alcobaça ${ }^{44}$, sin duda, el mismo citado por Erce $^{45}$.

pero está muy presente en la iconografía italiana. Para una visión actualizada de la historiografía sobre las diferentes versiones de este complejo milagro véase R. PLÖTz, "Res est nova et adhuc inaudita. Índice de motivos y evolución literario-oral del relato del milagro del peregrino que fue rescatado de la horca", en P. CAUCCI Von SAUCKEN (Coord.): San Giacomo e l'Italia, Centro Italiano di Studi Compostellani, Perugia, 2005, pp. 531-573.

${ }^{36}$ Más de la mitad del texto dedicado al retablo se centra en la aportación de datos y fuentes para conocer la hagiografía de las escenas en él representadas. En el caso de la figura del caballero y su caballo cubiertos de conchas, el autor realiza un larguísimo soliloquio sobre el origen, sea de la representación sea de las conchas, ofreciendo datos que van desde la historia natural hasta la teología que, a pesar de su indiscutible valor para el estudio iconógico, son accesorias para el tema que ahora nos interesa (M. DE ErCE JimÉnez, Prueva evidente cit., Parte II, Trat. II, Cap. I, p. 230,9 - p. 232, 24).

37 Por ejemplo un "libro mui antiguo escrito de mano en la libreria del Monasterio de S. Juan de los Reyes de Toledo señalado con la letra X.num.90 que es de las vidas, i milagros de los Santos, i en el capitulo 49 que se intitula de las Conchas de Santiago; se refiere largamente al sucesso, contenido en el retablo de Roma", también "un Himno del Breviario antiguo de Oviedo en la fiesta del Apostol de 25 de Iulio", y finalmente "un Santoral antiquísimo escrito de mano en pergamino en lengua Portuguesa mui cerrada, que se conserva en el Real Monasterio de Alcobaza en Portugal” (M. De Erce y JiméneZ, Prueva evidente cit., Parte II, Trat. II, Cap. I, p. 230, 8).

${ }_{38}$ M. Castellá Ferrer, Historia del Apóstol de Iesus Christo Sanctiago Zebedeo Patrón y Capitan General de las Españas, Madrid, 1610, libro II, cap. II, p. 123.

${ }^{39}$ H. OxeA, Historia del Glorioso Apóstol Santiago Patrón de España, de su venida a ella, y de las grandezas de sus iglesias y órdenes militares, Madrid, 1615, cap. 28, n. 2, 177-79.

40 Licenciado Molina, Historia de Galicia, 1550, cap. 28, n. 2, LXI.

${ }^{41}$ M. Martins, “A Traslação de S. Tiago", en Brotéria, LXXVI, 1963, n. ${ }^{\circ}$ 1, pp. 59-65; M. Martins, “As Vieiras dos Peregrinos de Compostela", en Brotéria, LXXVI, 1963, n. ${ }^{\circ}$ 2, pp. 164-174.

42 A. De Magalhaes Ribeiro da Cunha: “A Devoção popular a Santiago de Compostela em Portugal”, en Brigantia, XVII (1986), pp. 77-114.

${ }^{43}$ El milagroso suceso ocurrió en la costa norte portuguesa, en Bouças, actual Matosinhos, cuando un caballero portugués y su caballo emergieron del mar, salvos y cubiertos de conchas al paso de la nave de la Traslación de Santiago. La historia parece remitirnos a la fundación de alguna casa o dinastía y aparece recogida en numerosas fuentes portuguesas, de entre las cuales Mário Martins ha transcrito el relato más completo (M. MARTins, “As Vieiras cit.”, p. 169, pp. 169171). Cronológicamente la leyenda debe ser posterior al Liber Sancti Iacobi, que la ignora, pero en Portugal existen ya referencias de tiempos de Alfonso X que indican su popularidad a finales del siglo XIII (Ibidem, pp. 171-172).

${ }^{44}$ Se trata de un Santoral del monasterio de Alcobaça fechado en 1442/43, conservado en la Biblioteca Nacional de Lisboa bajo la colocación Códice Alcobacense n. ${ }^{\circ} 280$ (M. MARTins, “A Traslação cit.”, p. 61, n. 15; M. MARTins, “As Vieiras cit.", p. 169).

${ }^{45}$ Erce enumera un largo número de fuentes, entre ellas "un Santoral antiquísimo escrito de mano en pergamino 
Pero si la Prueva evidente se muestra particularmente rica para el estudio iconográfico, no lo es menos para el historiador del arte pues la minuciosidad del relato es tal, que nos permite conocer particulares esenciales a la hora de identificar los fragmentos originales del retablo desaparecido.

El autor señala el color de los ornamentos del caballo de la tabla central del banco, "un cavallo negro con silla, $i$ adorno rojo rico", la forma de las veneras que cubrían su cuerpo y el del caballero, "conchas mui perfectas pintadas de la haz, i enves", o el gesto de la madre del peregrino ahorcado, "i la madre en pie con los braços abiertos levantados admirada"

Ante la claridad de los datos expuestos y tras una atenta lectura del texto completo, creemos legítimo identificar la tabla de Camerino con la parte central de la predella del desaparecido altar de Araceli y a las pinturas de La Spezia y Pau -relacionadas por Federico Zeri y Andrea de Marchi con la de Las Marcas- con los laterales de la misma obra ${ }^{47}$.

Tras todo lo expuesto podemos concluir afirmando que el texto de Erce confirma la relación estilística entre las tres obras estudiadas y posibilita su atribución al pintor Giovenale de Orvieto, abriendo una puerta hasta ahora cerrada para la creación de un catálogo del artista. Asimismo, podemos aventurar que dicho texto podrá contribuir todavía en el futuro a la identificación de otras partes de la desmembrada pala de Araceli ${ }^{48}$, fragmentos en los que, sin duda, debió dividirse a su llegada al mercado artístico italiano ${ }^{49}$.

Fecha de recepción: 15-IX-2007

Fecha de aceptación: 17-I-2008

en lengua Portuguesa mui cerrada, que se conserva en el Real Monasterio de Alcobaza en Portugal" (M. DE ERCE JiménEZ, Prueva evidente cit., Parte II, Trat. II, Cap. I, p. 231, 8).

${ }^{46}$ Referencias similares se repiten a lo largo de todo el texto: al describir la nave de la traslación se nos dice que se veía "el cuerpo de Santiago sobre la popa rostro venerable abierta la barba"; en referencia a la posición del caballero de las conchas se indica cómo "Está hablando con los de la nave echando el braço por el cuello de uno de ellos como sustentandose en el"; de la reina Lupa se nos dice que "que muestra el trage, que era señora de alta alcurnia" y, poco después, al describir la llegada de los bueyes a su palacio, que "està à las ventanas admirandose de ello".

47 Dada su longitud no hemos podido transcribir todo el texto que Erce dedica al retablo, pero remitimos al lector a las páginas que creemos pueden servir de sólida base a nuestra hipótesis (M. DE ERCE JiMÉnEZ, Prueva evidente cit., Parte II, Trat. II, Cap. I, p. 229, 1 - p. 230, 8 / p. 232, 25 - p. 233, 33.

${ }^{48}$ Los fragmentos correspondientes a la tabla central con Santiago, San Esteban y San Lorenzo, el remate con la pintura de un Calvario y los seis santos de las dos alas laterales descritos en la nota 26.

49 Se convierte ahora en un trabajo pendiente el estudio de la llegada del retablo al mercado, algo que ya había señalado Federico Zeri en relación con la pequeña tabla del Museo Amedeo Lia, según el autor "già sul commercio di Roma e di Firenze” (F. ZerI: "Antonio Alberti da Ferrara. Miracolo cit.”, p. 25). 